Chapter 14

\title{
Using Hypnosis in the Treatment of Anxiety Disorders: Pros and Cons
}

\author{
Catherine Fredette, Ghassan El-Baalbaki, \\ Sylvain Neron and Veronique Palardy \\ Additional information is available at the end of the chapter \\ http://dx.doi.org/10.5772/53768
}

\section{Introduction}

In psychotherapy outcome research, many empirical studies have shown that cognitive behavioural treatments are efficacious for many disorders [1]. In a recent systematic review of 27 studies, Hofmann and Smits [2] show that cognitive behavioural therapy (CBT) has proven to be an unquestionably efficacious treatment for adult anxiety disorder when compared to both pharmacological and psychological placebos. However, they conclude that there was considerable room for improvement. Moreover, the high complexity and co-morbidity that is often found with anxiety disorders sometimes requires the use of two or more treatment methods that are flexible and adjustable to one other [3]. According to Kirsch, Lynn, and Rue [4] and Schoenberger [5], hypnosis can be integrated easily into current cognitive and behavioural interventions in clinical practice. Indeed, CBT and hypnosis share a number of aspects that render their combination natural; for example, imagery and relaxation, which are found in both techniques [6]. Hypnosis has been used effectively in a variety of medical settings (surgery, dentistry, chronic pain management, labour etc.) and several studies report its efficacy in the treatment of anxiety disorders [7-13]. A recent systematic review of randomized controlled trials concludes that current evidence is not sufficient to support the use of hypnosis as a sole treatment for anxiety [14]. However, in a meta-analysis, Kirsch, Montgomery, and Sapirstein [15] found that the addition of hypnosis to CBT substantially enhanced the treatment outcome for several problems (anxiety, obesity, pain, etc.). The addition of hypnosis to CBT helps the patient in several aspects of therapy, such as the preparation for in-vivo exposure, imagery exposure, developing coping skills, and cognitive restructuring [6, 16-18]. Moreover, patients using hypnosis effectively develop a better sense of self-efficacy, which is known to enhance self-regulation and is linked to lower psychologi- 
cal distress and better quality of life. Hence, hypnosis is worth exploring as an additional tool to improve traditional CBT.

In this chapter, we offer a comprehensive review of the literature regarding the use of hypnosis in the treatment of anxiety disorders. We will present evidence that supports its use or not as an adjunct treatment to CBT, also known as cognitive-behavioral hypnotherapy $(\mathrm{CBH})$. We will also present evidence that does not justify its use as an independent treatment for anxiety disorders. Due to the amount of research on Post-Traumatic Stress Disorder (PTSD) and hypnosis, the reader will notice that a lot of the information will be related to PTSD. We will conclude by giving a simple guideline for practitioners interested in developing and using hypnosis as an adjunctive therapeutic tool in their practice.

\section{Description and definition}

Although under different names and applications, hypnosis has been depicted, described and documented in ancient civilizations (e.g. Egyptians, Greeks, Chinese, Indians, Sumerians, Persians and others) and was mostly used by healers. In his book Ash Shifa (Healing), Ibn Sina (Avecenna) wrote about the mind-body relationship and accepted the reality of hypnosis, naming it "al Wahm al-Amil" [19]. He differentiated it from sleep and described the impact of imagination on sensation and perception [20]. More recently, the British physician James Braid [1795-1960], who is recognized for conducting many research studies and experiments on hypnotic phenomena, coined the words neurypnology and neuro-hypnosis [21, 22]. In fact, he observed his patients while in trance and concluded that they were in a "nervous sleep." The Greek word for sleep is hypnos [21]. These terms were quickly transformed into the word hypnosis. Hypnosis lost its appeal with the rise of psychoanalysis during the first half of the 20th century [23]. Indeed, after a short interest in the practice of hypnosis, Freud abandoned and rejected the idea [21]. As a valid form of psychotherapy, hypnotherapy only regained its popularity with the advent of the First and Second World Wars [23]. During this time, psychiatrists were faced with a new disease, called shell shock or war fatigue, and used hypnosis as a way to relieve the symptoms [21]. Today, this disorder is known as PTSD. Subsequently, the modern study of hypnosis began to flourish. Throughout the years, hypnosis has been represented in various ways, whether good or bad, and many popular misconceptions around this phenomenon remain [22]. Indeed, people under hypnosis are sometimes viewed as robots who do things that they would not normally do [22]. Even though individuals under hypnosis are more prone to suggestions, they still remain in control of what they say and do [24]. In fact, despite the perception that experiences under hypnosis often contain automatic or involuntary actions, hypnotised patients ultimately act in congruity with their goals and in accordance with their points of view [25]. Another mistaken belief is that hypnosis is not real. However, recent scientific studies (e.g. brain imaging studies) go beyond these mainstream conceptions and expose the true nature of hypnosis and its possible uses [25]. 
Burrows, Stanley, and Bloom [26] describe hypnosis as a technique that induces, through relaxed and focused attention, an elevated state of suggestibility. During this state, reduction in critical thinking, reality testing and tolerance of reality distortion allow the person to experience different phenomena (vivid imagery, drug free anaesthesia, drug free analgesia, and so on) that might otherwise be hard to attain [26]. Contrary to common perceptions, hypnosis is a natural phenomenon which people experience in a lighter way several times a day [27]. Daydreaming, being so absorbed by a book or movie that you do not hear someone calling your name or absent-mindedly driving past an expressway exit are all examples of shallow hypnotic states [27]. According to the division 30 of the American Psychological Association (APA), a procedure becomes a hypnotic one when the following two components are present: an introduction in which a person is told that suggestions for imaginative experiences will come, and the first suggestion, which functions as the induction [22]. Examples of suggestions during the introduction include: "I am going to ask you to imagine some changes in the way you think and feel. Is that ok? Let's see what happens" [22]. The formulation of hypnotic suggestions is different from other types of suggestions (e.g. placebo, social influence), given the fact that it requests the patient to participate [22].The first suggestion might come directly after the introduction and is usually a suggestion to close the eyes, move the arm or hand or alter perception [22]. Given that there are many types of hypnotic suggestions, standardized scales of suggestibility can be applied before someone undergoes formal hypnotherapy to see how suggestible the person is to all kinds of hypnotic suggestions [28]. During ideomotor suggestions, a certain action, such as arm levitation occurs automatically without awareness of volitional effort by the person [28]. Challenge suggestions occur when the hypnotised person is unable to execute an act that is ordinarily under voluntary control such as bending an arm [28]. Cognitive suggestions also can be used to create various cognitive or visual distortions such as pain reduction, selective amnesia, and hallucinations [28]. These different types of suggestions were characterized by Hilgard [29] as the domain of hypnosis.

Hypnotic experiences take place in the realm of imagination of the person under hypnosis [30]. However, it is interesting to note that hypnotic mental imageries and ordinary ones do not have the same experiential qualities [30]. Indeed, the construction of a mental imagery is both intentionally and consciously created, whereas imaginary experiences under hypnosis are generally involuntary [30]. People are suggested or informed about an image and it naturally comes to them. This difference seems to be supported by the fact that neurocognitive activations differ from normal and hypnotic imaginary experiences [31]. Another characteristic of hypnotic experiences, including the ideomotor ones, is that they are cognitive in nature [30]. Indeed, participants simply experience alterations in cognitive processes such as perception and memory. People differ in their abilities to experience hypnosis and it might be that some hypnotic responses require specific underlying abilities that are not shared by everyone, or that many individual components might be needed to experience a hypnotic phenomenon [32]. The ability to dissociate, cognitive flexibility, susceptibility to suggestions, fantasy proneness, and imaginative abilities were identified as possible traits that make an individual more amenable to experience hypnosis [33-36]. 


\section{Theories of hypnosis}

Hypnotic techniques became popular long before people knew what they were and how they worked. In the past, theorists viewed hypnosis as an altered state of consciousness or trance, but the quest to find evidence of this presumed state remained fruitless [28]. Indeed, it was discovered that people can respond in a similar yet slightly diminished way to nonhypnotic suggestions, suggesting that hypnosis is just another normal experience [28]. Moreover, since people under hypnosis are able to execute a full range of behaviours, theories needed to be able to encompass all of these aspects [28]. Due to the failure to explain such phenomena, several theories of hypnosis were developed, such as the psychoanalytic theory, the reality-testing theory, and more recently, the cold control theory and the discrepancy-attribution theory [21, 37, 38]. However, toward the end of the 20th century, two theories stood out as the most researched and influential ones: the dissociative theory and the sociocognitive theory.

Dissociative theories were first developed based on speculations about links between hypnosis and the phenomenon of dissociation [28]. Although a clear definition of dissociation is lacking, the first proponent of the dissociation theory described it as a split in the subunits of mental life, resulting in one or more parts left out from conscious awareness and voluntary control [39]. The neodissociative theory, developed by Hilgard, posits that hypnotic behaviours are produced by a "division of consciousness into two or more parts" [28] in which "part of the attentive effort and planning may continue without any awareness of it all" (p.2, 40]. Additionally, these subsystems are coordinated by a higher-order executive system, the 'executive ego' [39]. According to this theory, hypnosis alters the functioning of the executive ego, which tricks the mind about what is really going on. For example, when someone is asked to raise their arm under hypnosis, the executive ego might be responsible for the movement; however, because the awareness component of this has been separated into another part, this appears as an involuntary act to the hypnotised person [28].

Akin to dissociative theories, sociocognitive theories reject the idea that hypnosis requires an altered state of consciousness [41]. In fact, the same individualized social and cognitive variables that shape complex social behaviours are thought to determine hypnotic responses and experiences [41]. These variables are (a) a positive experience (attitudes, expectations, beliefs) with hypnosis in general, (b) good motivation to respond to suggestions, (c) clear indications that signal how to respond to hypnotic suggestions, and (d) implicit or explicit instructions in which to become absorbed or to imagine suggestions provided by the hypnotist. It is thought that when all of these variables are working together in a given individual, the person is under hypnosis [25]. Moreover, sociocognitive theories state that responses under hypnosis are goal-directed and that hypnotised people continue to act according to their aims and values, just as they ordinarily behave according to a socialized role [42]. Finally, rather than being attributed to an altered state of mind, the enhanced responses seen in people under hypnosis are merely a reflection of increased motivation and expectations [42]. 
Beyond differences and resulting controversy steaming from the dissociative and sociocognitive theory perspectives, new findings from psychophysiological and brain imaging studies have allowed the scientific community to support the hypothesis that experiences under hypnosis are "genuine" [24]. Indeed, studies demonstrated that there are distinctive patterns of activation (anterior cingulate cortex and frontal cortical areas) attributable to hypnosis and that these patterns comprise mechanisms used in other familiar cognitive tasks (focused attention, imagination, absorption) [24, 31]. Furthermore, there are specific psychophysiological correlates for suggested experiences [24, 31]. Some studies demonstrated that there is a qualitative distinction between neurocognitive activations that occur when people are asked to imagine certain images under hypnosis and in ordinary conditions [31]. Also, the hypnotic experiences appear to create brain states closer to the real experience, a phenomenon corroborated by the subjective reports of individuals [31]. Finally, brain imaging and psychophysiological studies might also enrich our understanding of the respective contribution of the social context, the subject's aptitudes, expectations, and intrasubjective experience of hypnotic phenomena.

\section{The clinical use of hypnosis}

\subsection{Medical conditions}

\subsubsection{Hypnosis alone}

Thus far, the value of hypnosis has already been recognized for many physical and medical conditions. Indeed, in 1996, the National Institute of Health Technology Assessment Panel Report considered hypnosis as a viable and effective solution to treat pain associated with cancer and many other chronic pain conditions [43]. It was even found that in certain conditions, the degree of analgesia resulting from hypnosis matched or even exceeded that provided by morphine [43]. These findings are supported by the results of Montgomery, DuHamel, and Redd's[44] meta-analytic review, which found that $75 \%$ of the people experienced pain reduction due to hypnosis, and these reductions were found in both a clinical and a healthy population. In their review of the literature, Neron and Stephenson [45] also present evidence on the effectiveness of hypnotherapy for emesis, analgesia, and anxiolysis in acute pain. Montgomery et al. [46] found that when compared to empathic listening, presurgery hypnosis was more effective in reducing pain intensity and pain unpleasantness for breast cancer patients. In addition to reducing the pain associated with cancer, hypnosis was also found to effectively reduce the affective morbidities (anxiety, discomfort, and emotional upset) associated with the medical procedures [46-48], as well as reduce fatigue [46, 49], sleep problems [49], nausea [46] and the quantity of medication needed [46]. Similar results (reduction in pain, anxiety and medication and better satisfaction) were found for plastic surgery patients [50], severe burn care patients [51], women giving birth [52], breast biopsy patients [53] and patients undergoing dental procedures [54]. Hypnosis also served as a sole anaesthetic ingredient 
for thousands of surgeries [43]. Other medical conditions that have been found to be responsive to hypnosis are preoperative preparations for surgery, a subgroup of patients with asthma, dermatological disorders, irritable bowel syndrome, hemophillia, post-chemotherapy nausea and emesis (Pinnell \& Covino(2000) cited in 43). Of note is that in the medical environment, clinical hypnosis is provided as an adjunct to medical treatment. There is usually no time for multiple sessions based on skills acquisition and homework. Intervention is often provided at bedside, or in preparation and during medical procedures away from the usual office-based psychotherapy setting. The goal of care is often symptom relief and comfort during the medical procedure and not psychological therapeutic change, which is typically the end point of psychotherapy. Hypnosis is used because it is efficacious but most importantly it is practical (short: minimal practice, no homework or assignments; portable: self-hypnosis) ${ }^{1}$.

\subsection{2. $C B H$ As an adjunct to $C B T$}

Kirsch et al. [15] reported substantial effect sizes for problems such as weight loss, pain, anxiety, and insomnia. More specifically, it was found to be particularly effective for the treatment of obesity $[15,56]$. Indeed, long-term weight loss was maintained at followups, which is an issue for most people who gain their weight back soon after losing it [15]. In their review of the literature, Chambless and Ollendick [57] even identified hypnosis (in conjunction with CBT) as an empirically supported therapy for obesity, along with headaches and irritable bowel syndrome [57]. A study done with women suffering from chronic breast cancer pain revealed that cognitive hypnotherapy or $\mathrm{CBH}$ was effective not only in reducing pain, but also in decreasing pain over time as the cancer progressed [58]. As for cigarette smoking, many studies assessing the use of hypnosis as an adjunct to cognitive-behavioural interventions found good results [59], with the rate of abstinence varying from 31 to $91 \%$ at the end of treatment and 31 to $87 \%$ around the threefour month follow-ups [56]. However, these results should be interpreted with caution, as some research demonstrated considerable limitations such as the exclusive use of self-reports, small sample sizes, a lack of differentiation between hypnosis and relaxation techniques and no clear definition of cigarette smoking [56]. More recently, some studies using more reliable approaches showed promising results in the use of hypnosis for cigarette smoking. Indeed, results indicate that after treatment, at three month, six month and 12 month follow-ups, more participants in the hypnosis group were abstinent [60,61]. Rather than using $\mathrm{CBH}$, these studies either compared hypnosis to behavioural treatment or to a waiting-list control group. Hypnosis appears to be a promising avenue for many physiological and psychological problems but most importantly, hypnosis is a cost-effective alternative procedure [43]. However, as Schoenberger's review [62] indicates, more rigorous

1 Flory \& Lang provide examples and data supporting this type of hypnotic intervention used as a flexible and practical tool to alleviate pain, anxiety, and treatment side effects while potentially reducing the need for sedation and stabilizing the vital signs [55]. 
methodologies as well as more studies comparing specifically the added benefit of hypnosis to CBT are needed to determine its real effects.

\subsection{Anxiety disorders}

\subsubsection{Social anxiety disorder}

The essential feature of social anxiety disorder (SAD) or social phobia is an important and persistent fear or worry about social and performance situations [63]. Social phobia can be divided into two types: generalized, in which individuals fear most social situations (e.g. having a conversation, facing authority, speaking in front of people and so on); and specific, when individuals only fear one particular situation (e.g. eating in front of people). According to different studies, the prevalence of SAD ranges from three to $13 \%$ of the population. In their review of five meta-analyses that looked specifically at the treatment of SAD, Rodebaugh, Holaway, and Heimberg [64] found that CBT appears to provide benefits for adults diagnosed with SAD, with modest to large effect sizes when compared to waiting-list control, as well as moderate to large effect sizes from pre to post-treatment.

Hypnosis as a sole treatment. To our knowledge, there is only one randomized controlled trial testing the use of hypnosis as a sole treatment for social phobia. In early attempts to view the potential of hypnosis to treat social anxiety, Stanton [65] randomized 60 adults seeking help for handling their anxiety. Anxiety levels were assessed by the Willoughby Questionnaire. The author compared a hypnotic procedure consisting of positive suggestions and mental imagery to another group that listened to quiet music (movements from Mozart symphonies) and to a control group. Both experimental groups met in their respective groups for 30 minutes for three weeks. At the end of treatment, both experimental groups experienced a significant reduction in their anxiety, whereas the control group saw minor changes in their anxiety levels. Moreover, the reduction for the hypnosis group was larger. Finally, the therapeutic gains were maintained for the hypnosis group only at six month follow-ups. Although these results were encouraging, this study presented many limitations such as the fact that there was no statistical calculation of the difference between the hypnosis and music groups and that the validity of the instrument was not presented. One case report also indicated that hypnosis was useful in treating social phobia [66]. Although hypnosis was used as a sole treatment, the author pointed out that the patient had experience with typical phobia treatments such as systematic imaginal and in-vivo exposure and that this familiarity might have contributed to the successful outcome.

CBH. Schoenberger, Kirsch, Gearan, Montgomery, and Pastyrnak [67] conducted a randomized controlled study on public speaking anxiety in which they compared the efficacy of CBT to the same therapy combined with hypnosis and a waiting-list control group. The experimental treatments included cognitive restructuring and in-vivo exposure. The hypnosis component consisted of replacing relaxation training by hypnotic inductions and suggestions [67]. In terms of self-report measures of public speaking 
anxiety, both experimental treatments produced a reduction in anxiety compared to the control group. As for the subjective and behavioural measures of fear, only the hypnotic group differed significantly from the control group. These measures were taken by a blind observer during a impromptu speech that participants gave in front of two observers. Finally, the mean effect sizes calculated across the dependant measures revealed a significant difference between the two experimental groups in favor of the hypnotic treatment (mean effect for the nonhypnotic treatment is 0.80 standard deviation and 1.25 standard deviation for the hypnotic treatment, $t(5)=3.75, p<.05)$ [67].

\subsubsection{Specific phobias}

A phobia is characterized by a marked and persistent fear prompted by the presence or anticipation of an encounter with a specific object or situation [63]. This situation can create a sensation of panic, somatic manifestations of anxiety, fainting or even trigger a panic attack in the phobic person. According to the DSM-IV-TR [63], there are 5 subtypes of phobias: animal type, natural environment type, situational type, blood-injection-injury type, and other type, which includes all phobias that do not fit in the previous categories. The lifetime prevalence rate varies from 7.2 to $11.3 \%$. CBT procedures (including in vivo-exposure and systematic desensitization) are considered the treatments of choice for specific phobias [68]. Even though these techniques apply to most phobias, certain ones require specific adaptation such as the applied tension technique for blood-injury-injection phobias [68].

Hypnosis as a sole treatment. We found two randomized controlled studies that utilized hypnosis as a stand-alone treatment for specific phobias. In the first one, Hammarstrand, Berggren, and Hakeberg [69] compared a group of women with dental phobias using two types of experimental treatments: a behavioural treatment based on psychophysiological principles, and hypnotherapy. The psychophysiological treatment consisted of progressive relaxation, videos of dental scenes and biofeedback training. As for the hypnotherapy, the participants were told to imagine different dental scenes, which corresponded to the videos of the psychophysiological group, and received suggestions. In addition, a control group who received general anaesthesia was added. Unlike the rest of the participants, this group was not randomized. The results showed that only the psychophysiological group experienced a significant reduction in anxiety. However, no significant difference between the two experimental and control groups was found. It should be noted that out of the 22 participants in this study, only 13 completed the treatments (eight in the psychophysiological treatment, five in hypnotherapy) and thus the sample was too small to draw real conclusions. Moreover, since the control group was not randomized, there is the possibility that these participants were different from the other two groups. In an exploratory study of four people suffering from specific phobias (i.e. fear of flying, snakes, driving, and heights), Llobet [21] assessed the effectiveness of group hypnotherapy. The hypnotherapy consisted of imaginary exposure with the use of the "magic bubble technique "2, as well as the age regression technique ${ }^{3}$ [21]. The author 
stated that behavioural techniques expose patients to the avoided stimuli in a "here and now" context [21]. Although these techniques have been proven efficacious, therapy should employ self-exploration in order for patients to understand their unique conscious and unconscious processes [21]. Age regression hypnotherapy can thus solve this problem [21]. Results of this study indicated that all participants saw their anxiety reduced in a significant way. Even though the participants' anxiety increased slightly at the two-week follow-up, participants still experienced on average a $56.46 \%$ decrease compared to their baseline score. The benefits of the group therapy might have been enhanced if it was combined with other well-recognized methods for treating phobias - a focus for future research.

$\mathrm{CBH}$. As for the integration of hypnosis with CBT or behavioural protocols for specific phobias, we found several case reports and case studies and only one randomized controlled trial. Recently, Forbes [71] compared the relative effectiveness of systematic desensitization with hypnosis to the same treatment with relaxation in the management of animal phobias. His results showed that patients in the hypnosis group enjoyed greater anxiety reduction than the other group. Finally, case studies also corroborated the effectiveness of $\mathrm{CBH}$ for driving phobia [72], animal phobia [73], and airplane phobia [74]. This evidence tends to support the use of $\mathrm{CBH}$ as an effective therapy for different types of phobias.

\subsubsection{Panic disorder with or without agoraphobia}

The main feature of Panic Disorder with or without Agoraphobia (PD/A) is the presence of recurrent, unexpected panic attacks, accompanied by persistent concerns about having other panic attacks, worry about the possible implications or consequences of panic attacks, or a significant behavioural change related to the attacks [63]. As for panic attacks, they are discrete periods of intense fear or discomfort that are accompanied by both physical and cognitive symptoms such as heart palpitations, hyperventilation, dizziness, a fear of losing control or going crazy, depersonalization and so on. People who suffer from PD sometimes develop agoraphobia, which is an anxiety related to being in places or situations in which escape might be difficult or impossible and help difficult to receive. In community samples, rates vary between one and two percent, although higher rates $[3.5 \%)$ were found in some studies (75]. When treating PDA, both the Canadian Psychological Association (CPA) and the APA recognize CBT as the first line of treatment [76]. Indeed, efficacious and robust treatment effects of this therapy have been verified across a variety of treatment settings for extended follow-up periods.

Hypnosis as a sole treatment or in conjunction with other non-cognitive and behavioural techniques. The use of hypnotic techniques to treat PD/A was successfully identified in some case reports [77-79]. Hypnotic techniques such as age regression, hypnoanalysis ${ }^{4}$ [77], ego-strength-

\footnotetext{
2 In order to render exposures less distressing, patients under hypnosis are suggested to imagine themselves in a magic bubble when they revisit their feared object or situation, which acts as a protection.

3 During age regression, the person is guided back in time to a past experience in order to relive it, or the person can also be suggested to remember the experience in a here-and-now as vividly as possible [70].
} 
ening suggestions [78], and the use of medication in conjunction in one case [79] led most patients to become panic free. However, no controlled trial studies could be found on hypnosis alone.

$\mathrm{CBH}$. In the only controlled trial study on the efficacy of $\mathrm{CBH}$ in treating PDA, Dyck and Spinhoven [80] demonstrated that a combined therapy (self-hypnosis and exposure) was not superior to exposure alone in terms of time spent by agoraphobics walking on a prescribed route. In this case, the hypnotic technique employed was imaginary exposure plus suggestions from the therapist consisting of successful encounters with the feared situation (prescribed route). One problem with this study is that it used a cross-over design (exposure alone and then combined/ combined followed by exposure alone) and thus the eventuality that patients still continued to use hypnosis during the exposures alone cannot be ruled out. Thus, reservations must be kept in mind with regard to these latter results. Interestingly, the authors also found that preference for treatment shifted toward the combined treatment as the study went on [80]. Positive results for CBH were demonstrated in many case reports [18, 81, 82]. Indeed, hypnosis was found to enhance CBT protocols by facilitating exposures to both the symptoms of panic and situational anxiety. Moreover, it also was found to be successful in conjunction with Rational Emotive therapy (RE).

\subsubsection{Generalized anxiety disorder}

People who suffer from Generalized Anxiety Disorder (GAD) experience excessive and hardly controllable worry and anxiety most of the time. Contrary to some other anxiety disorders where the anxiety is focused on a specific event or thing (e.g. specific phobia), GAD individuals worry about different situations and activities. Many individuals also develop somatic symptoms such as muscle tension, nausea, and sweating. In community samples, approximately three percent of the population will develop GAD [63]. As for the treatment of GAD, traditional narrative reviews and meta-analyses have consistently found that CBT and applied relaxation are the most efficacious treatments [83].

Hypnosis as a sole treatment. Recently, a study investigated hypnosis as an alternative method for CBT in the treatment of GAD [27]. The hypnosis component was comprised of suggestions involving the lessening of anxiety. Based on the Beck Anxiety Inventory (BAI) scores of 60 patients, the author stated that there was evidence of hypnosis being as effective as CBT in the treatment of GAD [27]. These results were derived from the archived records of a local licensed mental health therapist's private practice. Although these results are positive, the patients were not randomized to the treatments but rather assigned to treatment based on their own desire to receive hypnotherapy or CBT. It is thus safer to say that hypnosis was as effective as CBT for patients who believed in and wished to be treated with hypnotherapy. Also, since this was a retrospective study, many aspects such as the number of sessions, and the integrity of therapy could not be controlled for.

4 Hypnoanalysis is a mix of hypnosis and psychoanalytic techniques 
$C B H$. In a pilot randomized controlled study of 10 patients, Allen [84] assessed the comparable efficacy of a treatment incorporating CBT, hypnosis, and biofeedback to a waiting-list control group. All patients in the experimental group demonstrated a reduction in both trait and state anxiety. Most of them (four out of five) even obtained post-test state anxiety scores below the normative range. As for the control group, their anxiety remained at a clinically significant level [84]. CBH also came out as a successful aid in the treatment of GAD, as demonstrated by Baker's [85] case report.

\subsubsection{Obsessive-compulsive disorder}

Obsessive-Compulsive Disorder's (OCD) main features are recurrent obsessions and/or compulsions that are so severe that they are time-consuming and/or cause distress to the person. Obsessions may be persistent ideas, thoughts, impulses or images that can be related to many different topics such as contamination, religion, symmetry and repeated doubts. As for compulsions, they are repetitive behaviours or mental acts that people perform in order to diminish the anxiety associated with their obsessions. The estimated lifetime prevalence of OCD is $2.5 \%$ [63]. In a recent review of the literature, Podea, Suciu, Suciu, and Ardelean [86] concluded that CBT is an effective treatment for OCD, that it is at least as effective as medication and that it demonstrates good benefits at follow-ups.

$\mathrm{CBH}$. So far, hypnosis has occupied a relatively restricted role in the treatment of OCD [87] and this is reflected in the few numbers of studies on this topic. Indeed, no well controlled studies on the efficacy of $\mathrm{CBH}$ have been completed so far to see the additive effect of hypnosis to CBT [88]. Rather, the hypnosis literature only contains descriptions of clinical work done with a minimal number of patients and a series of case studies usually unaccompanied by measurable data. Still, as a combination to CBT, hypnosis was found to be efficacious in many case reports and one case study [88-92]. For example, because his patient did not respond to CBT and medication, Frederick [88] developed an intervention in which CBT and hypnoanalysis were incorporated. The hypnosis part was mainly aimed at the resolution of the dissociative symptoms. Other authors used hypnosis during exposures (e.g. exposure-response prevention, flooding) in order to enhance its effect, relieve anxiety and ameliorate the patients' affect regulation [90-92]. Very recently, Meyerson and Konichezky [87] presented three single-case reports in which hypnoticallyinduced dissociation (HID) combined with CBT protocols was successfully used in order to treat patients with OCD. According to Yapko [93], HID is the ability to split a fully and unified experience into many different components, while amplifying awareness of one part and diminishing awareness of the others. For example, some patients report that they cannot recognize themselves without their disorder. HID can thus be used to help the person dissociate him or herself from the disorder and amplify their feeling of experiencing life without the disorder. 


\subsubsection{Post-traumatic stress disorder}

In the DSM-IV-TR [63], PTSD is described as the development of characteristic symptoms after an individual is exposed to an extreme traumatic stressor (A1). The traumatic event must put at risk the physical integrity of the individual or others and the person's response must involve intense fear, helplessness, or horror (A2). The characteristic symptoms of PTSD include (B) stress and hyperarousal, (C) persistent avoidance of situations or reminders of the trauma and (D) vivid experiences of being back in the midst of the traumatic event, which are often referred to as a flashback. Finally, (E) these symptoms must last for at least one month. If the time is less than that, the diagnostic is labelled as Acute Stress Disorder (ASD). PTSD lifetime prevalence rates are approximately eight percent. In high risk populations such as veterans, these rates may rise to as high as 30\% [94]. In terms of treatment, variations of CBT protocols such as cognitive processing therapy (CPT) and prolonged exposure are known to effectively treat PTSD symptoms [95].

Among all of the anxiety disorders, the addition of hypnosis to CBT in the treatment of PTSD is the most studied. This interest has been triggered by factors such as the evidence that PTSD patients seem to be more highly hypnotisable when compared to the general population and other patient populations [96-98]. Butler, Duran, Jasiukaitis, Koopman et al. [99] developed a diathesis-stress model of dissociation to explain this phenomenon which is that "highly hypnotisable/dissociative people would be more likely to develop posttraumatic/ dissociative conditions rather than other psychiatric conditions". Evidence in support of this model are the fact that higher scores on hypnotisability scales are associated with avoidance symptoms, which is a core aspect of PTSD [96] as well as with better therapeutic success [100]. However, research is needed to exclude the possibility that it is the development and maintenance of PTSD that create a state of high hypnotisability. Moreover, clinical findings seem to suggest that there is a similarity in phenomenology between PTSD symptoms and the experience of hypnosis [101]. For example, during hypnosis, the person is entirely focused and absorbed into the suggestions and this absorption is also evidenced in PTSD sufferers, who sometimes focus so intensely on their traumatic memories that they are able to create physical and emotional responses. Another common factor is the phenomenon of dissociation, which can occur both during and after the trauma. Finally, both PTSD and hypnosis are experiences in which the person is hyper-responsive to both their environment (social, physical cues) and internal cues [101]. Because traditional interventions are mostly aimed at targeting the core symptoms of PTSD, the interest in hypnosis was also prompted by the fact that as a flexible form of treatment, it might be able to target important symptoms such as sleep and dream disturbance, pain, and emotional and anxiety withdrawal problems associated with traumas $[100,102]$.

Hypnosis as a sole treatment or in conjunction with relaxation training. A recent randomized controlled study tested the hypothesis that hypnosis could help relieve the cluster of hyperarousal symptoms in PTSD, in a group of women who had experienced sexual trauma [103]. This study compared the use of a hypnotic induction (Elkins Hypnotisability Scale) to a standard care intervention, which was a combination of supportive counselling, CBT, interpersonal therapy, and solution-focused technique [103]. Following the ini- 
tial induction, a hypnotic induction recording for subjects in the treatment group was given to use at home over a period of one week. The author reported a statistically significant decrease in hyperarousal symptoms, general anxiety, and difficulty concentrating for the hypnotic group [103]. However, participants did not fall under the clinically significant line, and on many measures there was no significant difference between the control and treatment group. Some noticeable limitations of this study were that even though the groups were randomized, some of the baseline symptoms of the hypnosis group were more severe than that of the control group, which might explain the small differences between the two groups on some measures at the end of treatment. [103]. Even though it was the study's goal to create a short treatment, it came out that one week was probably too short of an interval to see the real effects of hypnosis and reach clinically significant results. It would have been interesting to see the added benefit of hypnosis to the standard treatment over a longer period of time. Moreover, in this study, there was minimal use of hypnosis. Indeed, the hypnotic induction did not include any suggestions to treat aspects of PTSD.

As part of their symptoms, PTSD sufferers often complain about sleep problems [17]. Some studies indicated that hypnosis can be helpful in reducing time to sleep onset in a group of individuals with chronic insomnia [104, 105]. A meta-analysis of 59 outcome studies also demonstrated that the short-term effects of hypnosis (one-two months) and relaxation training were comparable to the effects of short-term drug therapy and that the long-term outcomes even surpassed the drug therapy in certain instances [106]. Abramowitz, Barak, BenAvi, and Knobler [107] studied a group of chronic combat-related PTSD sufferers who experienced sleep problems even though they received supportive therapy and serotonin reuptake inhibitors (SSRIs). The participants had difficulty falling asleep as well as maintaining sleep and reported night terrors. The authors compared the efficacy of two weeks of one-and-a-half hour hypnotherapy sessions with the drug therapy Zolpidem to see the effects on PTSD symptoms and sleep problems. They found that in addition to see a reduction in the major PTSD symptoms, the hypnotic group reported better sleep quality, fewer awakenings, and less morning sleepiness.

$\mathrm{CBH}$. There are many recent instances of case studies and reports that describe the success of hypnosis in conjunction with CBT for traumas associated with industrial accidents [108-110], motor-vehicle accident [111], sexual abuse and rape trauma [112-114], spouse abuse-related trauma [101, 114-116] and assault-related trauma [117]. For example, Degun-Mather [118] [119] reported the success of hypnosis in conjunction with CBT in two cases of patients suffering from different traumas (childhood and war). Hypnotherapy was used in order to activate and reconstruct the traumatic memories. On a larger scale, Brom, Kleber, and Defares [120] compared the effectiveness of four psychotherapeutic methods for the treatment of PTSD in 112 patients: hypnotherapy based on behavioural techniques, trauma desensitization, psychodynamic treatment, and a waiting-list control group, and determined that the treatment groups were significantly lower in trauma-related symptoms than the control group. However, the authors of the original study reported that there was still a lot of similarity between the three treatment conditions which could be due to similarities in the be- 
haviours of the therapists, which they did not measure directly. No statistical measures were presented to compare the active treatment groups. In their systematic review, Coelho, Canter, and Ernst [14] reanalyzed the data and found that on some measures (STAI-S, STAI$\mathrm{T}$, IES), the hypnotic group obtained statistically better results compared to the other treatment groups both at post-test and follow-up. Bryant et al.'s [121] randomized study compared the effectiveness of CBT, hypnosis and CBT, and social counselling for trauma survivors who suffer from ASD. The rationale behind their study was that hypnotic techniques might be able to breach dissociative symptoms of ASD [121]. A hypnotic induction was thus given right before imaginal exposure, in an attempt to ease the emotional processing of the traumatic memories [121]. Their results indicated that at post-treatment and follow-ups (six months, three years), fewer patients in the CBT (2.11\%) and hypnosis with CBT group $\left(4.22 \%{ }^{5}\right)$ met criteria for PTSD [121]. Also, hypnosis with CBT resulted in fewer re-experiencing symptoms than CBT alone at post-treatment, but this difference was not found at followup [121]. Even though these results are positive, the authors used hypnosis in only one aspect of their therapy (imaginary exposure). Hypnosis has many functions and is exploitable in many parts of therapy (which will be described in details below) and thus a broader application of it might have generated more additive gains and yielded clearer results. Moreover, the literature on hypnosis and PTSD is filled with examples of how hypnosis can be used specifically in the treatment of PTSD, so that its benefits can be enhanced. For further reading, see Lynn et al. [17] and Degun-Mather [119].

Recently, a new hypnotic technique called hypnotherapeutic olfactory conditioning (HOC) showed promising potential in the treatment of PTSD. Based on CBT protocols, HOC is a technique that helps patients create new olfactory associations in order to surmount anxieties and dissociative states [102]. More precisely, it is the "development, under hypnosis, of a positive olfactory association which allows the patient to regain control of their symptoms, especially when they were created by olfactory stimuli" (p.317 102). This technique is based on the notion that the sense of smell has the ability to create vivid memories due to the particular position of the olfactory bulb in the brain [102]. In an exploratory study of three individuals suffering from needle phobia, panic disorder and PTSD respectively, Abramowitz and Lichtenberg [122] found a marked reduction in the symptoms, as attested by the rating scales and reduction in the use of medication. In a prospective study testing HOC with 36 patients suffering from chronic PTSD, results demonstrated significant reductions in symptoms, as assessed by the Impact of Events Scale (IES-R), Beck Depression Inventory, and Dissociative Experiences Scale [102]. The gains were maintained at six month and one year follow-ups. In this study, the authors did not compare the direct added benefits of HOC to standard protocols. However, the fact that most patients had already been in therapy for a mean time of more than two years and that baseline symptoms presented significant psychopathology indicates that HOC was able to provide additional benefits to the therapy. Still, replication studies are needed for HOC.

5 results for 3 year follow-up. 


\subsubsection{Other anxiety-related problems}

Hypnosis as a sole treatment. A 1978 study looked at the difference between two non-pharmacological interventions in the treatment of what was then called "anxiety neurosis". The two treatments were either a meditational relaxation technique comprised of muscle relaxation and concentration on inner breathing and stillness, or a self-hypnosis treatment, also comprised of muscle relaxation and suggestions to send tingling feelings and light to the parts of the body where anxiety symptoms were manifested [123].The participants were tested on levels of hypnotisability and were then separated into two groups: medium-high hypnotisable subjects and low hypnotisable subjects. Then, the participants in each group were randomized to one of the two experimental treatments. Although more participants in the hypnotic group improved according to the Hamilton Anxiety Rating Scale, the results indicated that there was essentially no difference between the two techniques in terms of therapeutic efficacy [123]. However, participants in the medium-high group, independently of the type of treatment, significantly improved on the psychiatric assessment and demonstrated a decrease in their average systolic blood pressure [123]. One major limitation of this study is that at the beginning, the authors randomized 69 people to the four treatment conditions, however, 37 of them did not complete the protocol. Thus, in addition to providing no results on the drop-outs, the benefits of randomization cannot be assumed in this study. Moreover, the hypnosis treatment was very similar in content to the meditational group, which can explain the minute difference between the two. Stanton [124] randomly assigned a group of 40 students to either a self-hypnosis training group or a control group, which consisted of discussions on ways to reduce test anxiety. The participants were matched on sex and anxiety scores. After two sessions and at a six month follow-up, anxiety scores were significantly reduced for the hypnotic group only. More recently, O'Neill, Barnier, and McConkey [125] compared self-hypnosis training with progressive relaxation in a group of stressed, anxious, and worried patients. At a one month follow-up, both groups indicated significant improvement on the Beck Anxiety Inventory (BAI-State and Trait) but no significant difference was found between these two groups on the BAI. However, the hypnosis group surpassed the relaxation group on cognitive changes and perceptions of treatment efficacy [125]. Indeed, the hypnosis patients reported superior expectations of the success of therapy [125]. A closer look at the procedures revealed that the content of the instructions given to both groups were very similar. These results seem to indicate that the simple fact of defining certain aspects of therapy hypnosis provided confidence and better expectation in patients [16]. Finally, in an attempt to determine the effectiveness of hypnosis on test anxiety, Hyman [126] randomized 21 participants to a hypnotic-induction only group, a postsuggestion hypnotic group or a control group. The participants received only one session of hypnosis and the post-hypnotic suggestions consisted of suggestions for reduction of test anxiety [126]. The results showed that directly after the inductions, there was no significant difference between the three groups, as evidenced by the Test Anxiety Inventory (TAI). At one month follow-up though, a significant difference was observed between the post-hypnotic group and the control group in terms of anxiety. The post-hypnotic suggestion group was also the only group who experienced a significant decrease in test anxiety over time (between post and follow-up assessments). Although the sample size of this study was very 
small, this seems to indicate that post-hypnotic suggestions might be one of the active ingredients of hypnosis $\mathrm{CBH}$.

A study comparing the effects of two hypnotic procedures (imagery and cognitive restructuring under hypnosis versus hypnotic induction only) with two control groups (attention placebo and no treatment) on the treatment of test anxiety supports the idea that the combination of hypnosis and CBT offers more therapeutic gains [127]. Indeed, results indicate that while the induction-only group had more improvements than the two control groups, only the group receiving imagery and cognitive restructuring under hypnosis obtained significant results on anxiety and academic performance [127].

\subsubsection{Summary and conclusions on the clinical use of hypnosis}

To date, except for PTSD, there is a very small number of randomized controlled studies assessing the impact of $\mathrm{CBH}$ for the treatment of anxiety disorders, which limits the conclusions that can be drawn about its external validity. However, the results presented above still indicate that $\mathrm{CBH}$ is a promising treatment modality. Indeed, in addition to demonstrating its efficacy as a complete intervention to reduce anxiety symptoms, all studies that compared the additive effect of hypnosis found positive results, except for one. As stated before, this study used a cross-over design which might explain the lack of superiority for the combined group (exposure and hypnosis). Also, Mellinger [128] and Scrignar [91] reported the success of hypnosis as a valuable adjunct to render exposure practices more viable. Finally, using non-leading methods, Degun-Mather [118] reported the successful use of hypnosis to transform the fragmented memories of a war veteran who suffered from chronic PTSD and dissociative fugues into a complete narrative, leading to re-appraisal and re-structuring of the trauma. As for the evidence supporting hypnosis as a stand-alone treatment, results are mixed. Indeed, some of the case reports and studies presented above found positive results $[21,27]$. On the other hand, in 2003, the STEER [129] looked at four randomized controlled trials of hypnotherapy as a sole therapy for anxiety, coming to the conclusion that there was insufficient evidence regarding the efficacy of hypnotherapy and that it did not appear to be more effective than other treatments. In their conclusion, the authors of the STEER report also mentioned that the general quality of all studies was unsatisfactory. All of them presented major methodological flaws, such as a lack of established questionnaires, no use of imagery or suggestions during hypnosis, small sample sizes and no clear indications of qualification of competence of the therapists. This again renders it difficult to draw firm conclusions. More recently, a systematic review of controlled trial studies revealed that hypnosis as a sole treatment for anxiety was not superior than control conditions (waiting list controls, contact controls, or other non-standard treatments) [14], and though it is a powerful supportive tool, using it as a therapy by itself is an error [130]. Research on clinical hypnosis should reflect the clinical practice in psychotherapy, [56] and thus hypnosis should be viewed and studied as an adjunct to commonly used and recognized techniques. In fact, hypnotic technique can directly reinforce CBT strategies by helping patients to control and regulate the anxiety as well as the cognitive and attentional processes characteristic of many 


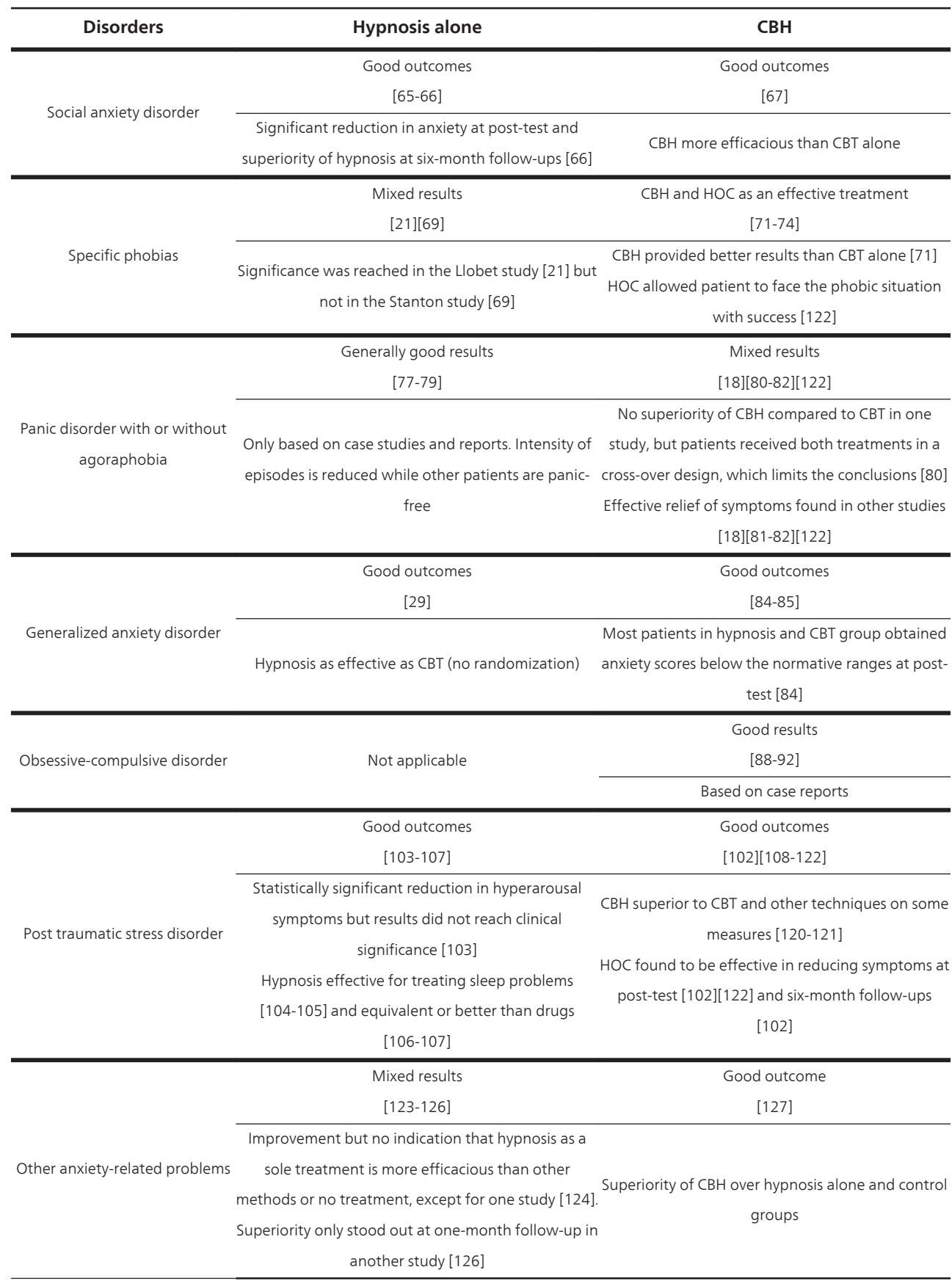

\section{Table 1.}


anxiety disorders [87]. One point to note, however, is that the boundaries between hypnosis as a stand-alone treatment and as an adjunct are sometimes unclear, as some people view a hypnotic induction followed by suggestions as CBT in itself [14]. For a summary table of the data presented above, see table 1.

\section{Guidelines and benefits of the application of hypnosis to CBT protocols}

\subsection{How to integrate hypnosis to CBT components}

As a psychosocial treatment, CBT has roots in both the cognitive and behavioural traditions and is based on the idea that our thoughts influence our feelings and behaviours [131]. Important components of CBT include relaxation training, exposure (both imaginal and invivo), cognitive restructuring, the building of coping skills, ego-strengthening, and selfefficacy. In the following, based on what several authors described (William, Bryant, Lynn and colleagues, Alladin, Degun-Mather), we will report a summary of how hypnosis can enhance each of these components $[6,16,17,23,119]$

Developing a good therapeutic alliance and motivation toward the therapy. The benefits of adding hypnosis to standard treatments of anxiety are manifold. The basis of therapy is the development of a good therapeutic alliance. The goal-directed and generally positive environment surrounding hypnosis may promote a better rapport with the therapist, as well as enhance treatment adherence [17]. For example, successful experiences of facing fears under hypnosis can foster trust in the hypnotherapist [23]. Moreover, positive views toward hypnosis might increase confidence in the effectiveness of therapy for certain patients [17].

Developing a sense of self-efficacy and heightened ego strengthening. Hypnotic techniques such as Ego strengthening are used to foster self-efficacy, self-esteem and self-assurance in patients. Self-efficacy provides a better quality of life, self-regulation and control and is one of the essential components in the successful treatment of anxiety disorders [23].

Self-control. Similarly, a great advantage of hypnosis is that it creates a feeling in the patients that they are in control of their difficulties, instead of being at the mercy of their symptoms [132]. Indeed, people learn to surmount their fears in trance and obtain cognitive reinforcement of their ability to cope [18].

Relaxation training. Relaxation techniques are an integral part of CBT as they help patients control their feelings of anxiety and tension [133]. For example, with the high level of arousal that PTSD patients tend to display, it can become difficult for them to fully participate in their therapy [17]. Many hypnotic techniques can serve to soothe patients and help them build personal resources [17]. For example, the patients can be taught relaxation techniques and learn the use of a safe place imaginary technique, which they can further practice by themselves using self-hypnosis [17]. Other relaxation techniques may include deep breathing, muscular relaxation and suggestions for relaxation. Hypnosis can be utilized easily in conjunction with all of these techniques. 
Imaginal exposure. Imagery is used by many CBT clinicians to facilitate anxiety reduction. The purposes of imagery are twofold: first, it can be utilized to induce relaxation by suggesting soothing and relaxing images to the patient. Secondly, imagery can be helpful for imaginal exposures, which is what is used to treat anxiety-provoking memories, images or thoughts. It consists of eliciting the patients to imagine their most feared memories or worst imagined outcome of feared situations and then to make them realize that their anxiety subsides even though they still think of the situation. By definition, hypnosis is an intense absorption into internal experiences that has the ability to create vivid images through increased body awareness, heightened suggestibility and a relaxed state [16, 21]. Also, suggestions under hypnosis can touch many aspects such as cognition, physical sensations and emotions [16]. Hypnosis can thus greatly enhance the emotional engagement of patients during exposure as well as make people experience their fears more intensely than with relaxation, which in turn could improve its efficacy [16, 21]. Hypnosis could also be used in the context of imagery rescripting therapy (IRT;23). As an imagery-based cognitive treatment, IRT employs exposure not for habituation but rather as a way for activating images, emotions, and beliefs associated with traumatic memories. Through the process of activation, the goal of this therapy is to modify and restructure the traumatic images, dysfunctional beliefs, attributions and schemas. Alladins' IRT [23] is comprised of four components: imaginal exposure, imaginal restructuring, self-calming and self-nurturing, and emotional linguistic processing. Within each of these components, well-known hypnotic techniques such as the bubble technique and the comforting the child technique can be used. For example, during the imaginal rescripting phase, the patient creates a mastery image during which the "survivor self" enters the traumatic scene to assist the "traumatized self". The integration of the split screen technique ${ }^{6}$ can be used to render the traumatic event more bearable. Finally, in addition to augmenting imagination, hypnosis facilitates both non-conscious and non-linguistic information processing that are often part of traumas [23].

In vivo-exposure. In-vivo exposure is an important element of therapy for all anxiety disorders; however, this experience can be very distressing and disorienting for certain patients, and can even lead to early dropouts [80,100]. Moreover, with patients suffering from a trauma, a degree of symptom stability, ego-strengthening and the capacity to tolerate emotionally charged imageries are required before beginning exposure therapy [17, 115]. Hypnosis can thus be used as an effective preparation tool for the expositions [17]. Indeed, hypnosis can help control and modulate the experience of patients and give them adequate tools to feel more secure and calm [100, 119]. For example, patients can learn, through self-hypnosis, to imagine a comforting and secure place in order to render their experience less distressing [17]. In addition, a patient who suffers from OCD can be instructed to touch objects which the person feels are contaminated while the hypnotherapist provides suggestions that "no harm will come to him" [132]. Through many imagined rehearsals of coping, resistant patients may become more confident in their ability to face in-vivo exposures. During exposure in PTSD, clinical psychologists must be careful not to re-traumatize the clients. 
Hypnosis can therefore help to prevent unnecessary exposure to too many traumatic events [119]. Indeed, indirect hypnotic safeguards such as ideomotor signalling for answering specific questions (e.g. Do you feel ready to regress to the past and address some event that is necessary for healing and which you feel able to cope with at present with help?) provide the hypnotherapist with the confirmation that the patient is able to embark on age-regression of the traumatic events [119].

Cognitive restructuring. Some of the early hypnotherapists already recognized the usefulness of hypnosis for cognitive restructuring [6]. Indeed, one important part of therapy is to teach patients to monitor and recognize their maladaptive thoughts. The increased suggestibility and reduced cognitive processing that accompany hypnosis make it a tool for rapid cognitive changes [133]. Hypnotherapy can be used to teach patients to replace their negative selfsuggestions (e.g." I'm not good enough to make a good presentation") with hypnotic suggestions ("I've done many of them and I've always had good feedback") that reduce their anxiety [6]. These suggestions also can be applied during self-hypnosis [6]. Hypnotic interventions also can help to strengthen patients' flexible thinking styles [134]. For example, it can help facilitate cognitive restructuring and re-appraisal of traumas; for example, through dissociation and self-distancing techniques, such as the split-screen technique, the imagination of a safe place, and the use of the "older" or "compassionate" self [17, 119]. A self-distance perspective is thought to promote insight and closure, as well as a reduction in rumination and distress [17]. With a self-distance perspective, people can come to see the situation from a different angle, which was impossible for them before as they focused on simply recounting their experience (and creating a whole lot of distressing symptoms). For example, with the use of the "compassionate self', a guilty PTSD patient can realize that he/she could not have done more and that the situation in question was out of his or her control. Another use of hypnosis is as a tool for memory integration, which can help promote cognitive restructuring [118].

Building coping skills. An important aspect of anxiety is its physical symptoms. For example, according to the DSM-IV [63], GAD is characterized by somatic symptoms such as muscle tension, irritability, insomnia and restlessness [135]. Moreover, according to cognitive-behavioural theories, $\mathrm{PD} / \mathrm{A}$ is based on the acquisition of the fear of physical sensations, especially those associated with the autonomic nervous system [136]. When PDA patients feel anxious, physical symptoms such as heart palpitations, headaches, and difficulty breathing can arise [18]. Building coping skills and self-efficacy under hypnosis is an effective way to control these symptoms [18]. Recently, autogenic training was found to produce significant reductions in blood pressure and pulse rate, which are often symptoms of anxiety [137]. According to Hammond [138], autogenic training is like a "structured German form of selfhypnosis" (p.264]. Hypnosis also can be utilized to help patients manage their physical responses to anxiety provoking stimuli so that they can dissociate somatic responses to psychological distress [135].

Another coping skill that patients can acquire is to learn to redirect their attention away from distressing cues. For example, in PTSD, arousal and avoidant symptoms are triggered by both internal and external cues associated with the memories of the trauma. Unfortunately, PTSD 
patients seem to be particularly distractible to these cues and the result is that their condition cannot improve. Lynn et al. [17] propose that hypnosis can facilitate attention control in these patients so that they can stop being absorbed by cues of traumatic memories. Indeed, they propose that if it is possible to suggest to a patient to enter a state of hypnosis, it is possible to suggest that this same patient experiences enhanced attention and concentration (p.322). Thus, people under hypnosis can learn not only how to focus their attention in the moment, but also how to switch their attention away from increasingly distracting cues [17]. The latter hypnotic attentional control learning can also be useful to help patients contend with their flashbacks [17]. However, Lynn et al. [17] also propose that this technique should also be accompanied with suggestions for increased tolerance to disturbing flashbacks.

Building of social skills. Anxiety disorders can create disturbances in interpersonal relationships or even be exacerbated by a lack of social competence [133]. The teaching of social skills is thus a common component of CBT protocols. With hypnosis, the patients can practice their new social skills in imaginal rehearsals [133]. On a different level, as explained by Alladin [23], early traumas created by abuse and neglect, for example, can affect people's internal working models or relational schemas. Moreover, these core relational schemas are sometimes relatively unresponsive to verbal information or the views expressed by the patients' relatives so that when different opinions are uttered, the patient will not believe them. In conjunction with a hypnotic technique such as age regression, reframing work can be done to change some of these core beliefs.

Overcoming resistance. According to Kraft [139], hypnosis can be used as a technique to counteract resistance to therapy and exposure that is sometimes found in agoraphobics, for example. Moreover, hypnotists can resort to indirect hypnotic suggestions to counteract patients' resistance to suggestions. For example, they can paradoxically instruct a patient to continue to resist to a given suggestion in order for this individual to get some control in the decision-making during the psychological intervention. The objective is to ultimately elicit compliance [23].

Behavioural modification. Another way that hypnosis can be useful is through the administration of post-hypnotic suggestions, which works by shaping the patients' behaviours and experiences after therapy [16]. Post-hypnotic suggestions are defined as instructions to a hypnotised person to show certain behaviours or have certain experiences after hypnosis [32]. For anxious individuals, suggestions can include to experience less anxiety during their daily routines, comply with therapy homework, employ coping strategies when faced with distressing situations or stimuli, and become aware of adaptive appraisals made during times of anxiety [16]. Furthermore, the hypnotherapist can make post-hypnotic suggestions that the patient will be able to deal with adverse situations with greater confidence [17]. According to Yapko [70], post-hypnotic suggestions are widely used in hypnotherapy. So far, there is little yet increasing empirical evidence of the efficacy of post-hypnotic suggestions. For instance, recently it was discovered that post-hypnotic suggestions were capable of simulating several clinical conditions such as blindness, amnesia, auditory hallucinations, conversion disorder paralysis, selected delusions, $[31,140]$ and neglect-like visual behaviours in healthy patients [141]. Moreover, with highly hypnotisable participants, post-hypnotic suggestions were used to reduce the automatic tendency to read printed words in a Stroop task [142, 143], and to reduce the Simon effect, which is the facilitation of lateralized responses, when they are executed in the same side of space as that 
of the stimulus [144]. The latter demonstrations thus support the clinical use of post-hypnotic suggestions to extend the achievements made during therapy.

Power of treatment. Finally, recent studies show that when used properly, hypnosis adds leverage to treatment and accelerates the recovery processes [18, 23, 27, 103]. According to Alladin [23], this is due to the fact that "hypnosis produces a syncretic cognition, which consists of a matrix of cognitive, somatic, perceptual, physiological, visceral, and kinaesthetic changes" (p.104).

\subsection{Hypnotisability assessment}

Different opinions remain as to whether or not levels of hypnotisability should be assessed before undergoing hypnotherapy [145]. These divergent opinions are based among other things, by the fact that some studies do report a link between levels of hypnotisability and treatment gains $[102,103]$ while others do not $[121,146,147]$. Moreover, researchers are facing some difficulties when trying to link hypnotisability with treatment outcomes. Indeed, one problem lies in the timing of the assessment [56]. When participants undergo a standard test of hypnotisability prior to their treatment, they are likely to infer conclusions about their own susceptibility to hypnosis. This could, in turn, influence their expectations toward the success of the treatment, which could ultimately affect their treatment outcome [56]. On the other hand, when hypnotisability levels are assessed after the treatment, the participants' experience during the treatment might then have an influence on subsequent levels of responsiveness under hypnosis [56]. On a more positive note, Lynn and Shindler [145] state that modern evaluation techniques have rendered possible the use of a good hypnotisability assessment. They also present the advantage of being able to evaluate a variety of factors (attitudes, beliefs, rapport with therapist, motivation to respond) that could influence the response to hypnosis, and to model the hypnotherapy techniques around it in order to augment the efficacy of the treatment [145]. Indeed, as it was stated before, considering participants' attitudes and expectations of hypnosis is crucial, as expectation of positive therapeutic outcome is more often than not predictive of improvement in treatment [148]. In term of hypnotisability levels displayed by participants, expectancies have also been demonstrated to play a major role [149]. Moreover, a good assessment is imperative to remove clients who are unsuitable candidates for hypnotherapy due to their conditions (e.g. patients who are more prone to psychotic decompensation, those with a paranoid level of resistance to being controlled) [145]. Needless to say, this evaluation goes beyond the simple use of formal scales of hypnotisability [145]. For guidelines on how to assess patients' level of hypnotisability, refer to Lynn and Shindler [145].

\subsection{Research on hypnosis}

There remains a long way to go before hypnosis as an adjunct to the treatment of anxiety disorders is considered a first-line treatment. Future research will need to conduct good quality randomized controlled trials for each of the anxiety disorders. Well-conducted multiple case studies from independent researchers also must be done to establish the validity of hypnosis as an adjunct to CBT. Studies must have adequate sample sizes so that good power can be achieved, and provide an intent-to-treat analysis in order to have better chances of finding conclusive results. They also need to have a clear detailed protocol for the hypnotic techniques used, 
for replication purposes. Moreover, as suggested by Lynn et al. [43], good descriptions of the population at hand permit replication and help in assessing the external validity of the results. Such descriptions should include the diagnostic procedures, patients' demographic and treatment history, use of medication, comorbid diagnoses, and tests administered [43]. According to Schoengerber [56], despite the difficulties met while assessing hypnotisability levels, good attempts should be made to do so. For example, the Stanford Hypnotic Susceptibility Scale-Form $\mathrm{C}$ (SHSSC) is considered a gold standard measure and a good individual measure, and the Waterloo adaptation of this scale, the WGSC, is good for group administration [62]. Furthermore, in order to avoid the possibility that disproportionate numbers of high hypnotisable participants end up in one group compared to another, researchers could randomly match or stratify participants in terms of their hypnotisability scores or at least report the hypnotic suggestibility of each group in terms of scores. These scores could then be used as covariates in statistical analyses if groups differ considerably on this variable [43]. As some studies seemed to indicate that the effect of adding hypnosis appeared or persisted in the long-term $[15,126]$, studies should include follow-up measures. In conclusion, in accordance with the Society for Clinical and Experimental Hypnosis, this chapter argues that hypnosis is a technique and not a type of therapy and that it should be used as a tool to augment the efficacy as well as the patients' understanding of CBT principles [43, 134].

\section{Conclusion}

Clinical hypnosis is a flourishing area of research that has so far demonstrated the usefulness of hypnosis in many domains, especially in the treatment of pain in the medical environment and during medical procedures [55]. According to Bryant [16], there is no doubt that hypnosis can ameliorate established means of treating anxiety disorders. However, more research needs to be conducted in order to provide the information necessary to establish hypnosis (added to CBT) as an empirically supported treatment for anxiety disorders. The lack of adequate studies on this topic points to the need for more rigorous randomized controlled investigations on the use of hypnosis for anxiety disorders. This chapter, as well as many other books and articles [16, 23, 94, 150] present many ways in which hypnosis can be added to CBT. Researchers who wish to study hypnosis can refer to these as guidelines.

William [6] pointed out that hypnotherapy does not need to prove that it is superior to other forms of treatment in order to have clinical value. Indeed, the goal of clinical psychology is to determine what treatments are working for which patients with which problems, and under what conditions (Lazarus, 1973; cited in 6). Moreover, as stated before, hypnosis is a very cost-effective method [43] that could represent in some cases, a rapid, non-addictive and safe substitute to the use of medication, which is particularly important given the current increase in health care costs and adverse economic conditions [138]. Another advantage of hypnotherapy is that it can be used easily outside the clinic under the form of self-hypnosis. Self-hypnosis is defined as the employment of hypnotic suggestions through self-talk or listening to a recording of hypnotic suggestions [16]. Contrary to popular belief, how intensely someone responds to hypnosis resides in the ability of the individual, rather than in 
the special skills of the hypnotist [16]. Thus, self-hypnosis is a viable solution to help maintain the skills that were acquired during therapy. Consequently, hypnosis seems to respect the principle of parsimony, one of the most popular principles of clinical psychology, by creating more rapid gains and enhancing the efficacy of CBT interventions. Indeed, clinical psychologists should always try to utilize the least complex and most efficacious mode of treatment first [138].

This chapter focused on the use of hypnosis in the treatment of adult anxiety disorders. It is important to note that hypnosis is a therapeutic tool that is suitable for child and adolescent therapy. Indeed, although most research on hypnosis focus on adults, the popularity of complementary and alternative forms of therapies has started to attract parents of children with different problems [151]. According to Saadat and Kain [151], hypnosis is a suitable therapy for children because in general, they are more hypnotisable than adults. This is thought to be due to their increased capacity and willingness to become absorbed in fantasy, play, and imagination [151]. Moreover, psychologists can easily design specific hypnosis goals and suggestions that are individualized to the child and respect a developmental psychopathology perspective [152]. As for adults, meta-analyses and overviews have demonstrated the efficacy of hypnotherapy in treating children medical conditions such as asthma, chronic and acute pain, along with procedure-related distress for cancer patients [3]. Hypnosis also has improved child behavioural conditions such as trichotillomania, thumb-sucking, enuresis, dysphasia and chronic dyspnea [151]. However, Huynh et al's. [3] review of the literature revealed no randomised or controlled trials on the use of hypnotherapy for children with psychiatric disorders. Still, a high number of case reports indicated that hypnotherapy can be useful in treating children with PDA, social and specific phobias, OCD, GAD, and PTSD [3]. However, as is the case for adult anxiety disorders, the addition of hypnosis to clinical practice for children and adolescents needs to be developed and studied further before it is recognized as an empirically supported treatment.

\section{Author details}

Catherine Fredette ${ }^{1}$, Ghassan El-Baalbaki ${ }^{1,2}$, Sylvain Neron ${ }^{2}$ and Veronique Palardy ${ }^{1}$

1 University of Quebec at Montreal, Quebec, Canada

2 Mcgill University, Quebec, Canada

\section{References}

[1] Chambless D, L., , Baker M, J., , Baucom H, D., , Beutler L, E , Calhoun K, S, CritsChristoph P, et al. Update on Empirically Validated Therapies, II. The Clinical Psychologist. 1998;51(1):3-16. 
[2] Hofmann SG, Smits JA. Cognitive-behavioral therapy for adult anxiety disorders: a meta-analysis of randomized placebo-controlled trials. J Clin Psychiatry. 2008;69(4): 621-32. Epub 2008/03/28.

[3] Huynh ME, Vandvik IH, Diseth TH. Hypnotherapy in child psychiatry: The state of the art. Clinical Child Psychology and Psychiatry. 2008;13(3):377-93.

[4] Kirsch I, Lynn SJ, Rhue JW. Introduction to clinical hypnosis. In: troduction to clinical hypnosis JWR, Lynn SJ, Kirsch I, editors. Handbook of clinical hypnosis. Washington, DC, US: American Psychological Association; 1993. p. 3-22.

[5] Schoenberger NE. Cognitive-Behavioral Hypnotherapy for Phobic Anxiety. Casebook of clinical hypnosis. Washington, DC, US: American Psychological Association; 1996. p. 33-49.

[6] William L, Golden. Cognitive hypnotherapy for anxiety disorders. American Journal of Clinical Hypnosis. 2012;54(4):263-74.

[7] Alladin A. Handbook of cognitive hypnotherapy for depression : an evidence-based approach. Philadelphia: Wolters Kluwer Health/Lippincott Williams \& Wilkins; 2007.

[8] Ambrose G, \& Newbold, G. A handbook of medical hypnosis; an introduction for practitioners and students (3d ed.). Baltimore: Williams and Wilkins Co.; 1968.

[9] Brann L, Owens J, Williamson A. The handbook of contemporary clinical hypnosis : theory and practice. Chichester, West Sussex, UK: John Wiley \& Sons; 2011.

[10] Burrows GD, Dennerstein L. Handbook of hypnosis and psychosomatic medicine. New-York1988.

[11] Lynn SJ, Kirsch I, Rhue JW. An introduction to clinical hypnosis. In: Lynn SJ, Rhue JW, Kirsch I, editors. Handbook of clinical hypnosis (2nd ed). Washington, DC, US: American Psychological Association; 2010. p. 3-18.

[12] Nash M, R, Barnier A, J. The Oxford handbook of hypnosis: Theory, research, and practice. New-York, NY, US: Oxford University Press; 2008.

[13] Van Pelt SJ. Medical hypnosis handbook. Hollywood, California: Wilshire Book Co; 1957.

[14] Coelho HF, Canter PH, Ernst E. The effectiveness of hypnosis for the treatment of anxiety: A systematic review. Primary Care \& Community Psychiatry. 2008;12(2): 49-63.

[15] Kirsch I, Montgomery G, Sapirstein G. Hypnosis as an adjunct to cognitive-behavioral psychotherapy: A meta-analysis. Journal of Consulting and Clinical Psychology. 1995;63(2):214-20.

[16] Bryant RA. Hypnosis and anxiety: Early interventions. The Oxford handbook of hypnosis: Theory, research, and practice. New York, NY, US: Oxford University Press; 2008. p. 535-47. 
[17] Lynn SJ, Malakataris A, Condon L, Maxwell R, Cleere C. Post-traumatic Stress Disorder: Cognitive Hypnotherapy, Mindfulness, and Acceptance-Based Treatment Approaches. American Journal of Clinical Hypnosis. 2012;54(4):311-30.

[18] Nolan M. Hypnosis to enhance time limited cognitive-behaviour therapy for anxiety. Australian Journal of Clinical \& Experimental Hypnosis. 2008;36(1):30-40.

[19] Haque A. Psychology from Islamic Perspective: Contributions of Early Muslim Scholars and Challenges to Contemporary Muslim Psychologists. Journal of Religion and Health. 2004;43(4):357-77.

[20] Sina I. Kitab ash-Shifa (book of Healing). Jan Bakos ed. Prague: http://www.muslimphilosophy.com/books/shifa2.pdf; 10271956.

[21] Llobet P. Group hypnotherapy in the treatment of specific phobias: An exploratory study [3324378]. United States -- California: Alliant International University, San Diego; 2008.

[22] Barnier A, J., Nash M, R. Introduction: a roadmap for explanation, a working definition. In: Nash M, R and Barnier, A, J, editor. The oxford handbook of hypnosis: theory, research, and practice. New-York: Oxford University Press; 2008. p. 1-20.

[23] Alladin A. Cogntive hypnotherapy: An integrated approach to the treatment of emotional disorders. Chichester, England: John Wiley \& Sons, Ltd.; 2008.

[24] Lynn S, J, Green JP. The Sociocognitive and Dissociation Theories of Hypnosis: Toward a Rapprochement. International Journal of Clinical and Experimental Hypnosis. 2011;59(3):277-93.

[25] Lynn SJ, Green JP. The sociocognitive and dissociation theories of hypnosis: Toward a rapprochement. International Journal of Clinical and Experimental Hypnosis. 2011;59(3):277-93.

[26] Burrows GD, Stanley R, Bloom PB. International handbook of clinical hypnosis. Chichester, New-York: Wiley; 2001.

[27] Huston TR. The effects of using hypnosis for treating anxiety in outpatients diagnosed with generalized anxiety disorder. US: ProQuest Information \& Learning; 2011.

[28] Kirsch I, Lynn SJ. Dissociation theories of hypnosis. Psychological Bulletin. 1998;123(1):100-15.

[29] Hilgard ER. The domain of hypnosis: With some comments on alternative paradigms. American Psychologist. 1973;28(11):972-82.

[30] Kihlstrom J, F. The domain of hypnosis, revisited. In: Press OU, editor. The oxford handbook of hypnosis: theory, research and practice. New-York, N-Y, US2008.

[31] Oakley DA, Halligan PW. Hypnotic suggestion and cognitive neuroscience. Trends in Cognitive Sciences. 2009;13(6):264-70. 
[32] McConkey KM. Generations and landscapes of hypnosis: Questions we've asked, questions we should ask. In: Barnier MRNAJ, editor. The Oxford handbook of hypnosis: Theory, research, and practice. New York, NY, US: Oxford University Press; 2008. p. 53-77.

[33] Wilson SC, Barber TX. The fantasy-prone personality: Implications for understanding imagery, hypnosis, and parapsychological phenomena. PSI Research. 1982;1(3): 94-116.

[34] Aikins D, Ray WJ. Frontal lobe contributions to hypnotic susceptibility: A neuropsychological screening of executive functioning. International Journal of Clinical and Experimental Hypnosis. 2001;49(4):320-9.

[35] Spanos NP, et al. The Carleton University Responsiveness to Suggestion Scale: Relationship with other measures of hypnotic susceptibility, expectancies, and absorption. Psychological Reports. 1983;53(3, Pt 1):723-34.

[36] Lynn SJ, Rhue JW. The fantasy-prone person: Hypnosis, imagination, and creativity. Journal of Personality and Social Psychology. 1986;51(2):404-8.

[37] Dienes Z, Perner J. Executive control without conscious awareness: The cold control theory of hypnosis. Hypnosis and conscious states: The cognitive neuroscience perspective. New York, NY, US: Oxford University Press; 2007. p. 293-314.

[38] Barnier A, J,, Mitchell C, J. Looking for the fundamental effects of hypnosis: . 35th Annual Congress of the Australian Society of Hypnosis (Scientific Program); Sydney, Australia2005.

[39] Woody EZ, Sadler P. Dissociation theories of hypnosis. In: Barnier MRNAJ, editor. The Oxford handbook of hypnosis: Theory, research, and practice. New York, NY, US: Oxford University Press; 2008. p. 81-110.

[40] Hilgard ER. Divided consciousness: Multiple controls in human thought and action. New-York: Wiley; 1986.

[41] Lynn SJ, O'Hagen S. The sociocognitive and conditioning and inhibition theories of hypnosis. Contemporary Hypnosis. 2009;26(2):121-5.

[42] Lynn SJ, Kirsch I, Hallquist MN. Social cognitive theories of hypnosis. In: Barnier MRNAJ, editor. The Oxford handbook of hypnosis: Theory, research, and practice. New York, NY, US: Oxford University Press; 2008. p. 111-39.

[43] Lynn SJ, Kirsch I, Barabasz A, Carden a E, Patterson D. Hypnosis as an empirically supported clinical intervention: The state of the evidence and a look to the future. International Journal of Clinical and Experimental Hypnosis. 2000;48(2):239-59.

[44] Montgomery GH, DuHamel KN, Redd WH. A meta-analysis of hypnotically induced analgesia: How effective is hypnosis? International Journal of Clinical and Experimental Hypnosis. 2000;48(2):138-53. 
[45] Néron S, Stephenson R. Effectiveness of Hypnotherapy with Cancer Patients' Trajectory: Emesis, Acute Pain, and Analgesia and Anxiolysis in Procedures. International Journal of Clinical and Experimental Hypnosis. 2007;55(3):336-54.

[46] Montgomery GH, Bovbjerg DH, Schnur JB, David D, Goldfarb A, Weltz CR, et al. A Randomized Clinical Trial of a Brief Hypnosis Intervention to Control Side Effects in Breast Surgery Patients. Journal of the National Cancer Institute. 2007;99(17):1304-12.

[47] Snow A, Dorfman D, Warbet R, Cammarata M, Eisenman S, Zilberfein F, et al. A randomized trial of hypnosis for relief of pain and anxiety in adult cancer patients undergoing bone marrow procedures. Journal of Psychosocial Oncology. 2012;30(3): 281-93.

[48] Lang EV, Berbaum KS, Faintuch S, Hatsiopoulou O, Halsey N, Li X, et al. Adjunctive self-hypnotic relaxation for outpatient medical procedures: A prospective randomized trial with women undergoing large core breast biopsy. Pain. 2006;126(1-3): 155-64.

[49] Jensen MP, Gralow JR, Braden A, Gertz KJ, Fann JR, Syrjala KL. Hypnosis for symptom management in women with breast cancer: A pilot study. International Journal of Clinical and Experimental Hypnosis. 2012;60(2):135-59.

[50] Faymonville ME, Mambourg PH, Joris J, Vrigens B, Fissette J, Albert A, et al. Psychological approaches during conscious sedation. Hypnosis versus stress reducing strategies: A prospective randomized study. Pain. 1997;73(3):361-7.

[51] Harandi AA, Esfandani A, Shakibaei F. The effect of hypnotherapy on procedural pain and state anxiety related to physiotherapy in women hospitalized in a burn unit. Contemporary Hypnosis. 2004;21(1):28-34.

[52] Landolt AS, Milling LS. The efficacy of hypnosis as an intervention for labor and delivery pain: A comprehensive methodological review. Clinical Psychology Review. 2011;31(6):1022-31.

[53] Montgomery GH, Weltz CR, Seltz M, Bovbjerg DH. Brief presurgery hypnosis reduces distress and pain in excisional breast biopsy patients. The International journal of clinical and experimental hypnosis. 2002;50(1):17-32. Epub 2002/01/10.

[54] Mackey EF. Effects of Hypnosis as an Adjunct to Intravenous Sedation for Third Molar Extraction: A Randomized, Blind, Controlled Study. International Journal of Clinical and Experimental Hypnosis. 2009;58(1):21-38.

[55] Flory N, Lang E. Practical Hypnotic Interventions During Invasive Cancer Diagnosis and Treatment. Hematology/Oncology Clinics of North America. 2008;22(4):709-25.

[56] Schoenberger NE. Research on hypnosis as an adjunct to cognitive-behavioral psychotherapy. International Journal of Clinical and Experimental Hypnosis. 2000;48(2): 154-69. 
[57] Chambless D, L., , Ollendick T, H. . Empirically supported psychological interventions: Controversies and evidence. Annual Review of Psychology. 2001;52.

[58] Elkins G, Johnson A, Fisher W. Cognitive hypnotherapy for pain management. American Journal of Clinical Hypnosis. 2012;54(4):294-310.

[59] Green JP. Hypnosis and the treatment of smoking cessation and weight loss. In: Kirsch I, Capafons A, Cardeña-Buelna E, Amigó S, editors. Clinical hypnosis and self-regulation: Cognitive-behavioral perspectives. Washington, DC, US: American Psychological Association; 1999. p. 249-76.

[60] Carmody TP, Duncan C, Simon JA, Solkowitz S, Huggins J, Lee S, et al. Hypnosis for smoking cessation: A randomized trial. Nicotine \& Tobacco Research. 2008;10(5): 811-8.

[61] Elkins G, Marcus J, Bates J, Rajab MH, Cook T. Intensive Hypnotherapy for Smoking Cessation: A Prospective Study. International Journal of Clinical and Experimental Hypnosis. 2006;54(3):303-15.

[62] Schoenberger NE. Hypnosis in the treatment of women with anxiety disorders. Healing from within: The use of hypnosis in women's health care. Washington, DC, US: American Psychological Association; 2000. p. 45-64.

[63] APA. DSM-IV-TR. Washington, DC: American Psychiatric Association; 2000.

[64] Rodebaugh TL, Holaway RM, Heimberg RG. The treatment of social anxiety disorder. Clinical Psychology Review. 2004;24(7):883-908.

[65] Stanton HE. A comparison of the effects of an hypnotic procedure and music on anxiety level. Australian Journal of Clinical \& Experimental Hypnosis. 1984;12(2): 127-32.

[66] Rogers J. Hypnosis in the treatment of social phobia. Australian Journal of Clinical \& Experimental Hypnosis. 2008;36(1):64-8.

[67] Schoenberger NE, Kirsch I, Gearan P, Montgomery G, Pastyrnak SL. Hypnotic enhancement of a cognitive behavioral treatment for public speaking anxiety. Behavior Therapy. 1997;28(1):127-40.

[68] Barlow DH, Allen LB, Basden SL. Psychological treatments for panic disorders, phobias, and generalized anxiety disorder. In: Gorman PENJM, editor. A guide to treatments that work (3rd ed). New York, NY, US: Oxford University Press; 2007. p. 351-94.

[69] Hammarstrand G, Berggren U, Hakeberg M. Psychophysiological therapy vs. hypnotherapy in the treatment of patients with dental phobia. European Journal of Oral Sciences. 1995;103(6):399-404.

[70] Yapko MD. Trancework: An introduction to the practice of clinical hypnosis (3th Ed.). New York, NY, US: Routledge/Taylor \& Francis Group; 2003. 528 p. 
[71] Forbes S. Relative effectiveness of imaginal exposure with and without hypnosis in the treatment of specific animal phobias. [Dissertation]. London: University College; 2007.

[72] Hill R, \& Bannon-Ryder, G. The use of hypnosis in the treatment of driving phobia. Contemporary Hypnosis. 2005;22:99-103.

[73] Kraft D, Kraft T. Use of in vivo and in vitro desensitization in the treatment of mouse phobia: Review and case study. Contemporary Hypnosis. 2010;27(3):184-94.

[74] Volpe G, E., \& Nash, R, M. The use of Hypnosis for Airplane Phobia With an Obsessive Character: a Case Study. Clinical Cases Studies. 2012;11(1):1-15.

[75] Stein MB, Roy-Byrne PP, Craske MG, Bystritsky A, Sullivan G, Pyne JM, et al. Functional Impact and Health Utility of Anxiety Disorders in Primary Care Outpatients. Medical Care. 2005;43(12):1164-70.

[76] McCabe RE, Gifford S. Psychological treatment of panic disorder and agoraphobia. In: Stein MMAMB, editor. Oxford handbook of anxiety and related disorders. New York, NY, US: Oxford University Press; 2009. p. 308-20.

[77] DelMonte MM. The use of hypnotic regression with panic disorder: A case report. Australian Journal of Clinical Hypnotherapy and Hypnosis. 1996;17(1):1-5.

[78] Iglesias A, Iglesias A. Awake-Alert Hypnosis in the Treatment of Panic Disorder: A Case Report. American Journal of Clinical Hypnosis. 2005;47(4):249-57.

[79] Wild AJ. Hypnosis as an adjunct in the treatment of panic disorder. Australian Journal of Clinical \& Experimental Hypnosis. 1994;22(2):109-17.

[80] Dyck RV, Spinhoven P. Does preference for treatment matter? A study of exposure in vivo with or without hypnosis in the treatment of panic disorder with agoraphobia. Behavior Modification. 1997;21(2):172-86.

[81] Stafrace S. Hypnosis in the treatment of panic disorder with agoraphobia. Australian Journal of Clinical \& Experimental Hypnosis. 1994;22(1):73-86.

[82] Singh AR, Banerjee KR. Treating panic attack with hypnosis in combination with rational emotive therapy--A case report. Journal of Projective Psychology \& Mental Health. 2002;9(2):105-8.

[83] Fisher PL. The Efficacy of Psychological Treatments for Generalised Anxiety Disorder? Worry and its psychological disorders: Theory, assessment and treatment. Hoboken, NJ, US: Wiley Publishing; 2006. p. 359-77.

[84] Allen BT. A design of a combined cognitive-behavioral, biofeedback, and hypnosis training protocol for the reduction of generalized anxiety disorder. US: ProQuest Information \& Learning; 1998.

[85] Baker H. Hypnosis for anxiety reduction and ego-enhancement. Australian Journal of Clinical \& Experimental Hypnosis. 2001;29(2):147-51. 
[86] Podea D, Suciu R, Suciu C, Ardelean M. An update on the cognitive behavior therapy of obsessive compulsive disorder in adults. Journal of Cognitive and Behavioral Psychotherapies. 2009;9(2):221-33.

[87] Meyerson J, Konichezky A. Hypnotically induced dissociation (HID) as a strategic intervention for enhancing OCD treatment. American Journal of Clinical Hypnosis. 2011;53(3):169-81.

[88] Frederick C. Review of Hypnotically facilitated treatment of obsessive compulsive disorder: Can it be evidence-based? American Journal of Clinical Hypnosis. 2007;50(1):97.

[89] Moore KA, Burrows GD. Hypnosis in the treatment of obsessive-compulsive disorder. Australian Journal of Clinical \& Experimental Hypnosis. 1991;19(2):63-75.

[90] Proescher EJ. Hypnotically facilitated exposure response prevention therapy for an OIF veteran with OCD. American Journal of Clinical Hypnosis. 2010;53(1):19-26.

[91] Scrignar CB. Rapid treatment of contamination phobia with hand-washing compulsion by flooding with hypnosis. American Journal of Clinical Hypnosis. 1981;23(4): 252-7.

[92] Kroger WS, Fezler WD. Hypnosis and behavior modification: Imagery conditioning. Oxford, England: J. B. Lippincott; 1976. xxv, 426 p.

[93] Yapko MD. Essentials of hypnosis. Philadelphia, PA, US: Brunner/Mazel; 1995. xv, $185 \mathrm{p}$.

[94] Lynn SJ, Cardeña E. Hypnosis and the Treatment of Posttraumatic Conditions: An Evidence-Based Approach. International Journal of Clinical and Experimental Hypnosis. 2007;55(2):167-88.

[95] Bomyea J, Lang AJ. Emerging interventions for PTSD: Future directions for clinical care and research. Neuropharmacology. 2012;62(2):607-16.

[96] Bryant RA, Guthrie RM, Moulds ML. Hypnotizability in acute stress disorder. The American Journal of Psychiatry. 2001;158(4):600-4.

[97] Spiegel D, Hunt T, Dondershine HE. Dissociation and hypnotizability in posttraumatic stress disorder. The American Journal of Psychiatry. 1988;145(3):301-5.

[98] Stutman RK, Bliss EL. Posttraumatic stress disorder, hypnotizability, and imagery. The American Journal of Psychiatry. 1985;142(6):741-3.

[99] Butler LD, Duran REF, Jasiukaitis P, Koopman C, et al. Hypnotizability and traumatic experience: A diathesis-stress model of dissociative symptomatology. The American Journal of Psychiatry. 1996;153(Suppl):42-63.

[100] Carden a E. Hypnosis in the treatment of trauma: A promising, but not fully supported, efficacious intervention. International Journal of Clinical and Experimental Hypnosis. 2000;48(2):225-38. 
[101] Poon MW-L. Using hypnosis with a battered women with post-traumatic stress disorder. Australian Journal of Clinical \& Experimental Hypnosis. 2007;35(1):63-74.

[102] Abramowitz E, G., Lichtengerb P. A New Hypnotic Technique for Treating CombatRelated Posttraumatic Stress Disorder: A Prospective Open Study International Journal of Clinical and Experimental Hypnosis 2010;58(3):316-28.

[103] Auringer ML. Clinical efficacy of a brief hypnotic intervention for hyperarousal symptoms in sexual trauma. US: ProQuest Information \& Learning; 2011.

[104] Stanton HE. Hypnotic relaxation and the reduction of sleep onset insomnia. International Journal of Psychosomatics. 1989;36(1-4):64-8.

[105] Stanton HE. Hypnotic relaxation and insomnia: A simple solution? Sleep and Hypnosis. 1999;1(1):64-7.

[106] Morin CM, Culbert JP, Schwartz SM. Nonpharmacological interventions for insomnia: A meta-analysis of treatment efficacy. The American Journal of Psychiatry. 1994;151(8):1172-80.

[107] Abramowitz EG, Barak Y, Ben-Avi I, Knobler HY. Hypnotherapy in the Treatment of Chronic Combat-Related PTSD Patients Suffering From Insomnia: A Randomized, Zolpidem-Controlled Clinical Trial. International Journal of Clinical and Experimental Hypnosis. 2008;56(3):270-80.

[108] Carter C. The Use of Hypnosis in the Treatment of PTSD. Australian Journal of Clinical \& Experimental Hypnosis. 2005;33(1):82-92.

[109] Jiranek D. Use of hypnosis in pain management and post-traumatic stress disorder. Australian Journal of Clinical \& Experimental Hypnosis. 2000;28(2):176-87.

[110] Willshire D. Trauma and treatment with hypnosis. Australian Journal of Clinical \& Experimental Hypnosis. 1996;24(2):125-36.

[111] Chan R. A case study of chronic post-traumatic stress and grief: Hypnosis as an integral part of cognitive-behaviour therapy. Australian Journal of Clinical \& Experimental Hypnosis. 2008;36(1):13-22.

[112] Desland M. Post-traumatic stress disorder. Australian Journal of Clinical \& Experimental Hypnosis. 1997;25(1):61-73.

[113] Ebert BW. Hypnosis and rape victims. American Journal of Clinical Hypnosis. 1988;31(1):50-6.

[114] Poon MW-1. Hypnosis for complex trauma survivors: Four case studies. American Journal of Clinical Hypnosis. 2009;51(3):263-71.

[115] Kwan PSK. Phase-oriented hypnotherapy for complex PTSD in battered women: An overview and case studies from Hong Kong. Australian Journal of Clinical \& Experimental Hypnosis. 2009;37(1):49-59. 
[116] Kwan P, S, K. Hypnosis in complex trauma and breast cancer pain: a single case study Contemporary Hypnosis. 2007;24(2):86-96.

[117] Moore M. Hypnosis and post-traumatic stress disorder. Australian Journal of Clinical \& Experimental Hypnosis. 2001;29(2):93-106.

[118] Degun-Mather M. The value of hypnosis in the treatment of chronic PTSD with dissociative fugues in a war veteran. Contemporary Hypnosis. 2001;18(1):4-13.

[119] Degun-Mather MD. The use of hypnosis in the treatment of post-traumatic stress disorder in a survivor of multiple childhood trauma. Contemporary Hypnosis. 1997;14(2):100-4.

[120] Brom D, Kleber RJ, Defares PB. Brief psychotherapy for posttraumatic stress disorders. Journal of Consulting and Clinical Psychology. 1989;57(5):607-12.

[121] Bryant RA, Moulds ML, Nixon RDV, Mastrodomenico J, Felmingham K, Hopwood S. Hypnotherapy and cognitive behaviour therapy of acute stress disorder: A 3-year follow-up. Behaviour Research and Therapy. 2006;44(9):1331-5.

[122] Abramowitz EG, Lichtenberg P. Hypnotherapeutic olfactory conditioning (HOC): Case studies of needle phobia, panic disorder, and combat-induced PTSD. International Journal of Clinical and Experimental Hypnosis. 2009;57(2):184-97.

[123] Benson H, et al. Treatment of anxiety: A comparison of the usefulness of self-hypnosis and a meditational relaxation technique: An overview. Psychotherapy and Psychosomatics. 1978;30(3-4):229-42.

[124] Stanton HE. Self-hypnosis: One path to reduced test anxiety. Contemporary Hypnosis. 1994;11(1):14-8.

[125] O'Neill LM, Barnier AJ, McConkey K. Treating anxiety with self-hypnosis and relaxation. Contemporary Hypnosis. 1999;16(2):68-80.

[126] Hyman BH. Hypnotic treatment of test anxiety. US: ProQuest Information \& Learning; 2005.

[127] Boutin GE, Tosi DJ. Modification of irrational ideas and test anxiety through rational stage directed hypnotherapy [RSDH]. Journal of Clinical Psychology. 1983;39(3): 382-91.

[128] Mellinger DI. The role of hypnosis and imagery techniques in the treatment of agoraphobia: A case study. Contemporary Hypnosis. 1992;9(1):57-61.

[129] Dent T. Hypnotherapy for anxiety disorders. Southampton: 2003.

[130] Godoy PHT, Araoz DL. The use of hypnosis in posttraumatic stress disorders, eating disorders, sexual disorders, addictions, depression and psychosis: An eight-year review (part two). Australian Journal of Clinical Hypnotherapy and Hypnosis. 1999;20(2):73-85. 
[131] Weems CF, Varela RE. Generalized anxiety disorder. In: Storch DMEA, editor. Handbook of child and adolescent anxiety disorders. New York, NY, US: Springer Science + Business Media; 2011. p. 261-74.

[132] Kraft T, Kraft D. The place of hypnosis in psychiatry: Its applications in treating anxiety disorders and sleep disturbances. Australian Journal of Clinical \& Experimental Hypnosis. 2006;34(2):187-203.

[133] Evans BJ, Coman GJ. Hypnosis with treatment for the anxiety disorders. Australian Journal of Clinical \& Experimental Hypnosis. 2003;31(1):1-31.

[134] Kellis E. Clinical hypnosis and cognitive-behaviour therapy in the treatment of a young woman with anxiety, depression, and self-esteem issues. Australian Journal of Clinical \& Experimental Hypnosis. 2011;38(2):155-65.

[135] Nanda R, Singh B. Hypnotherapy in management of generalized anxiety disorder. In: Sharma R, Palan BM, editors. Hypnosis: Psycho-philosophical Perspectives and Therapeutic Relevance: Concept Publishing Compagny; 2011.

[136] Barlow DH. Clinical Handbook of Psychological Disorders: A Step-By-Step Treatment Manual: Guilford Press; 2007.

[137] Kanji N, White A, Ernst E. Autogenic training to reduce anxiety in nursing students: Randomized controlled trial. Journal of Advanced Nursing. 2006;53(6):729-35.

[138] Hammond DC. Hypnosis in the treatment of anxiety- and stress-related disorders. Expert Review of Neurotherapeutics. 2010;10(2):263-73.

[139] Kraft D. Counteracting resistance in agoraphobia using hypnosis. Contemporary Hypnosis \& Integrative Therapy. 2011;28(3):235-48.

[140] Oakley DA. Hypnosis as a tool in research: experimental psychopathology. Contemporary Hypnosis. 2006;23(1):3-14.

[141] Priftis K, Schiff S, Tikhonoff V, Giordano N, Amodio P, Umiltà C, et al. Hypnosis meets neuropsychology: Simulating visuospatial neglect in healthy participants. Neuropsychologia. 2011;49(12):3346-50.

[142] Raz A, Kirsch I, Pollard J, Nitkin-Kaner Y. Suggestion Reduces the Stroop Effect. Psychological Science. 2006;17(2):91-5.

[143] Raz A, Fan J, Posner MI. Hypnotic suggestion reduces conflict in the human brain. Proceedings of the National Academy of Sciences of the United States of America. 2005;102(28):9978-83. Epub 2005/07/05.

[144] Iani C, Ricci F, Baroni G, Rubichi S. Attention control and susceptibility to hypnosis. Consciousness and Cognition: An International Journal. 2009;18(4):856-63.

[145] Lynn SJ, Shindler K. The Role of Hypnotizability Assessment in Treatment. American Journal of Clinical Hypnosis. 2002;44(3-4):185-97. 
[146] Bryant RA, Moulds ML, Guthrie RM, Nixon RDV. The Additive Benefit of Hypnosis and Cognitive-Behavioral Therapy in Treating Acute Stress Disorder. Journal of Consulting and Clinical Psychology. 2005;73(2):334-40.

[147] Wadden TA, Anderton CH. The clinical use of hypnosis. Psychological Bulletin. 1982;91(2):215-43.

[148] Kirsch I. Changing expectations: A key to effective psychotherapy. Belmont, CA, US: Thomson Brooks/Cole Publishing Co; 1990. xviii, 231 p.

[149] Barnier AJ, Dienes Z, Mitchell CJ. How hypnosis happens: New cognitive theories of hypnotic responding. In: Barnier MRNAJ, editor. The Oxford handbook of hypnosis: Theory, research, and practice. New York, NY, US: Oxford University Press; 2008. p. 141-77.

[150] Zahi A, Meyerson J. Application of hypnotic strategies sustained by a positive psychology orientation in treating OCD patients. Contemporary Hypnosis. 2010;27(3): 177-83.

[151] Saadat H, Kain ZN. Hypnosis as a therapeutic tool in pediatrics. Pediatrics. 2007;120(1):179-81.

[152] Kaiser P. Childhood anxiety, worry, and fear: Individualizing hypnosis goals and suggestions for self-regulation. American Journal of Clinical Hypnosis. 2011;54(1): 16-31. 
